\title{
p35/p25 Is Not Essential for Tau and Cytoskeletal Pathology or Neuronal Loss in Niemann-Pick Type C Disease
}

\author{
Janice L. Hallows, Robert E. Iosif, Rebecca D. Biasell, and Inez Vincent \\ Department of Pathology, University of Washington, Seattle, Washington 98195
}

Hyperactivation of the cyclin-dependent kinase 5 (cdk5), triggered by proteolytic conversion of its neuronal activator, p35, to a more potent byproduct, $\mathrm{p} 25$, has been implicated in Alzheimer's disease (AD), amyotrophic lateral sclerosis, and Niemann-Pick type C disease (NPC). This mechanism is thought to lead to the development of neuropathological hallmarks, i.e., hyperphosphorylated cytoskeletal proteins, neuronal inclusions, and neurodegeneration, that are common to all three diseases. This pathological ensemble is recapitulated in a single model, the npc-1 $\left(\mathrm{npc}^{-/-}\right)$mutant mouse. Previously, we showed that pharmacological cdk inhibitors dramatically reduced hyperphosphorylation, lesion formation, and locomotor defects in $n \mathrm{pc}^{-/-}$mice, suggesting that cdk activity is required for NPC pathogenesis. Here, we used genetic ablation of the $p 35$ gene to examine the specific involvement of $\mathrm{p} 35, \mathrm{p} 25$, and hence cdk 5 activation in NPC neuropathogenesis. We found that lack of p35/p25 does not slow the onset or progression or improve the neuropathology of NPC. Our results provide direct evidence that p35/p25-mediated cdk5 deregulation is not essential for NPC pathology and suggest that similar pathology in AD may also be cdk5 independent.

Key words: Alzheimer's disease; ALS; cdk5; hyperphosphorylation; spheroid; tauopathy

\section{Introduction}

Abnormal aggregates of neuronal cytoskeletal proteins are signatures of many human neurodegenerative diseases. Neurofibrillary tangles (NFTs) composed of hyperphosphorylated tau are observed in Alzheimer's disease (AD) and tauopathies (Lee et al., 2001). Axonal spheroids containing neurofilaments (NFs) and hyperphosphorylated tau are observed in motor neuron diseases such as amyotrophic lateral sclerosis (ALS) (Delisle and Carpenter, 1984) and tauopathies (Axelsson et al., 1984; Zhou et al., 1998). Curiously both NFTs and spheroids are produced concomitantly in Niemann-Pick type C disease (NPC), a fatal neurovisceral lipidosis with widespread neurodegeneration. Antigenically and ultrastructurally, the NFTs of NPC are similar to those of AD and tauopathies, and the spheroids of NPC resemble those of ALS and tauopathies (Bu et al., 2002b; Walkley and Suzuki, 2004). Thus, NFTs and spheroids may be produced by similar posttranslational mechanisms in these etiologically diverse conditions.

Received Nov. 9, 2005; revised Jan. 30, 2006; accepted Feb. 1, 2006.

This work was supported by National Institutes of Health Grant AG12721 (I.V.). We thank Drs. Peter Davies (Albert Einstein College of Medicine, Bronx, NY), Ki-Young Lee (University of Calgary, Calgary, Alberta, Canada), and Koichi Ishiguro (Mitsubishi Kasei Institute of Life Sciences, Tokyo, Japan) for the gifts of some antibodies used in these studies. We thank Dr. Min Zhang (Tongji Hospital, Tongji Medical College, Hubei, China) for her advice on the $n p \mathrm{c}^{-1-}$ mice.

Correspondence should be addressed to Inez Vincent at her present address: Centre for Molecular Medicine and Therapeutics, University of British Columbia, 950 West 28th Avenue, Vancouver, British Columbia, Canada V5A 4H4. E-mail: ivincent@cmmt.ubc.ca.

J. L. Hallows's present address: Centre for Molecular Medicine and Therapeutics, University of British Columbia, 950 West 28th Avenue, Vancouver, British Columbia, Canada V5Z 4H4.

R. E. losif's present address: Section of Restorative Neurology, Wallenberg Neuroscience Centre, Biomedicinskt Centrum A11, SE-221 84 Lund, Sweden.

DOI:10.1523/JNEUROSCI.4834-05.2006

Copyright $\odot 2006$ Society for Neuroscience $\quad$ 0270-6474/06/262738-07\$15.00/0
Cyclin-dependent kinase 5 (cdk5) is thought to be responsible for tau hyperphosphorylation in these diseases (Cruz and Tsai, 2004). Cdk5 is activated by p35 or p39, both of which may be cleaved to more stable and potent fragments, p25 and p29 (Cruz and Tsai, 2004). Elevated p 25 and cdk5 hyperactivation are seen in AD (Patrick et al., 1999), ALS (Nguyen et al., 2001), and NPC (Bu et al., 2002a). Efforts aimed at supporting a causal relationship between $\mathrm{cdk} 5 / \mathrm{p} 25$ and NFTs have relied on overexpression of cdk5, p35, or p25. Although some of these models have yielded positive results (Cruz et al., 2003; Noble et al., 2003), others have not (Ahlijanian et al., 2000; Takashima et al., 2001; Bian et al., 2002).

We tackled this relationship by using an alternative approach of inhibiting cdk5 activity in a natural model replicating neurodegenerative features of human disease, the $n p c^{-1-}$ mouse. This model arose because of spontaneous mutations in the $n p c l$ gene, the orthologous gene mutated in human NPC. The NPC1 locus encodes a late endosomal/lysosomal lipid transporter (Walkley and Suzuki, 2004), and NPC1 mutations lead to lipid accumulation in all body tissues, weight loss, mental retardation or dementia, and motor defects with ataxia. $n p c^{-/-}$mice recapitulate most features of human NPC. Although they do not develop the ultrastructural characteristics of human NFT (German et al., 2001), they do accumulate hyperphosphorylated tau in neuronal somata and form axonal spheroids. Tau from $n p c^{-/-}$mouse brain appears similar to $\mathrm{AD}$ or human NPC tau by multiple qualitative and quantitative criteria (Bu et al., 2002a,b). These similarities suggest that signaling mechanisms leading to tau modification in $n p c^{-/-}$mice may be similar to those in the human diseases.

In vivo approaches are necessary for unraveling neurodegenerative mechanisms in NPC, because primary neurons from $n \mathrm{pc}^{-/-}$mice fail to develop cytoskeletal pathology and survive 
Table 1. Antibodies

\begin{tabular}{|c|c|c|c|c|c|}
\hline Group & Antibody & Isotype & Epitope & Dilution & Source \\
\hline \multirow[t]{2}{*}{ cdk5 } & cdk5 (DC 17) & Mouse lgG1 & Full-length cdk5 & $1: 500$ & Santa Cruz Biotechnology (Santa Cruz, CA) \\
\hline & cdk5 (C-8) & Rabbit polyclonal & cdk5 $C$ terminus & $1: 100$ & Santa Cruz Biotechnology \\
\hline \multirow[t]{2}{*}{ p35/p25 } & p35 (C-19) & Rabbit polyclonal & p35 C terminus & $1: 100$ & Santa Cruz Biotechnology \\
\hline & $\mathrm{p} 25 \mathrm{~N}$ & Rabbit polyclonal & p25 N terminus & $1: 250$ & K. Ishiguro \\
\hline p39 & p39 & Rabbit polyclonal & p39 N terminus & 1:1000 & K.-Y. Lee \\
\hline \multirow[t]{7}{*}{ Tau } & TG5 & Mouse lgG1 & Total tau & $1: 10$ & I. Vincent \\
\hline & CP13 & Mouse lgG1 & Phospho-tau Ser-202 & $1: 10$ & P. Davies \\
\hline & PHF-1 & Mouse lgG1 & Phospho-tau Ser-396/404 & $1: 20$ & P. Davies \\
\hline & MC6 & Mouse lgG1 & Phospho-tau Ser-235 & $1: 10$ & P. Davies \\
\hline & CP10 & Mouse lgM & Phospho-tau Thr-231 & $1: 10$ & P. Davies \\
\hline & $\mathrm{CP} 22$ & Mouse lgM & Phospho-tau Thr-175 & $1: 10$ & P. Davies \\
\hline & PG5 & Mouse lgG3 & Phospho-tau Ser-409 & $1: 5$ & P. Davies \\
\hline Neuronal antigens & NeuN & Mouse lgG1 & Neuronal nuclei & $1: 230$ & Chemicon (Temecula, CA) \\
\hline
\end{tabular}

normally in vitro (Henderson et al., 2000). We previously assessed the role of cdk activity in NPC by intracerebroventricular treatment of $n p c^{-1-}$ mice with the pan-cdk inhibitors roscovitine or olomoucine (Zhang et al., 2004). These inhibitors attenuated cytoskeletal pathology and neurodegeneration, indicating an essential role for cdks in NPC.

Toward defining which cdk is involved and to determine specifically whether p25 is required, we crossed $n p c^{-/-}$mice with p35 null mice. Because p25 is a derived proteolytically from p35, p35 null mice also lack p25. Constitutive cdk5 depletion is lethal (Ohshima et al., 1996), but p35 null mice are viable (Hallows et al., 2003). Behavioral, biochemical, and immunohistochemical comparisons of wild-type $(w t), p 35^{-1-}, n p c^{-/-}$, and $n p c^{-/-}$, $p 35^{-1-}$ littermates show that lack of p35/p25 does not slow the development of NPC or inhibit tau phosphorylation or lesion formation.

\section{Materials and Methods}

Mice. Heterozygous BALB/cNctr-Npcl $1^{m I N} / \mathrm{J}\left(n p c^{+/-}\right)$mice were obtained from The Jackson Laboratory (Bar Harbor, ME) and maintained in our colony. $n p c^{+/-}$mice were crossed with $p 35^{+/-}$mice having a C57BL/6 background (Hallows et al., 2003) to obtain double heterozygous $\left(n p c^{+/-}, p 35^{+/-}\right)$mice in the mixed BALB/c/C57BL/6 background. Double-heterozygous mice were mated to obtain $w t, p 35^{-/-}, n p c^{-/-}$, and $n p c^{-1-}, p 35^{-/-}$genotypes in the mixed background, and all comparisons between these different genotypes were made using littermates. It should be noted that the mixed background did not have an observable effect on the basic phenotypic features of either $n p c^{-/-}$or $p 35^{-/-}$mice. A more direct test of the effects of the C57BL/6 background on the phenotype has been done by backcrossing the BALB/c line into the C57BL/6 strain (Walkley and Suzuki, 2004). The disease phenotype was similar except for an increase in spheroids in the fimbria. The comparisons made in the present study did not involve this region of brain. Other transgenic or knock-out mice in the C57BL/6 background have also been crossbred with the $n p c^{-/-} \mathrm{BALB} / \mathrm{c}$ mice, without any noticeable effect on NPC pathology (Erickson and Bernard, 2002). All protocols and housing were approved by the Animal Care and Use Committee at the University of Washington.

Genotyping was performed by PCR analysis of genomic DNA isolated from tail biopsies obtained at the time of weaning as reported previously [npc (Loftus et al., 1997); $p 35$ (Hallows et al., 2003)].

Eight- to 9-week-old male and female mice were killed by decapitation, and brains were quickly dissected and halved sagittally. One-half was flash frozen for biochemical analyses, and the other half were immersion fixed overnight in $4 \%$ paraformaldehyde at $4^{\circ} \mathrm{C}$, paraffin embedded, cut in $10 \mu \mathrm{m}$ sections, and mounted on slides for histological analyses.

Antibodies. Table 1 lists all antibodies used for Western blotting. The NF SMI32 and the ganglioside GM2 antibodies used for histology were from Sternberger Monoclonals (Lutherville, MD) and Matreya (State
College, PA), respectively. Subtype-specific secondary antibodies conjugated to HRP or biotin and streptavidin-HRP were from Southern Biotechnology (Birmingham, AL).

Western blotting. Western blotting was performed as reported previously (Hallows et al., 2003). Briefly, frozen brain halves were homogenized in buffer, and aliquots were stored at $-70^{\circ} \mathrm{C}$. Supernatants of brain homogenates were electrophoresed, transferred to nitrocellulose, and probed with the antibodies listed in Table 1. In all experiments, protein levels were normalized to the levels of the neuronal antigen neuronalspecific nuclear protein (NeuN). Two to three blots were run for each set of samples for each epitope examined $\left(n=6 \mathrm{wt}, 6 \mathrm{npc}^{-/-}\right.$, and $6 \mathrm{npc} \mathrm{c}^{-/-}$, p $\left.35^{-1-}\right)$.

In vitro kinase assays. Cdk5 was immunoprecipitated from $100 \mu \mathrm{g}$ of brain homogenates using $3 \mu \mathrm{g}$ of polyclonal antibodies (Table 1), and activity was assayed using histone $\mathrm{H} 1$ (Calbiochem, La Jolla, CA) as a substrate as reported previously (Hallows et al., 2003) $(n=4 w t, 4$ $p 35^{-/-}, 4 n p c^{-/-}$, and $\left.4 n p c^{-/-}, p 35^{-/-}\right)$.

Histology. Sagittal brain sections were sequentially incubated with the phospho-tau antibodies CP13 or paired helical filament-1 (PHF-1) at 1:10, the NF antibody SMI32 at 1:500, or the GM2 antibody at 1:100, biotin-conjugated secondary antibodies, streptavidin-HRP, and then $\mathrm{DAB}$ to visualize antibody binding, followed by hematoxylin and eosin (H\&E) counterstaining as reported previously (Hallows et al., 2003). For phospho-tau staining, sections were first incubated with $50 \mathrm{mM} \mathrm{NH}_{4} \mathrm{Cl}$ for $30 \mathrm{~min}$ before incubation with the primary antibody. Images were collected using an Olympus Optical (Thornwood, NY) DP10 digital camera attached to an Olympus Optical BX40 microscope $(n=3 w t, 3$ $p 35^{-1-}, 6 n p c^{-1-}$, and $\left.6 n p c^{-1-}, p 35^{-1-}\right)$.

Purkinje neuron and axonal spheroid quantitation. Sagittal brain sections $150 \mu \mathrm{m}$ lateral to midline from 8- to 9-week-old mice were incubated with the SMI32 antibody to NFs to visualize Purkinje neurons and axonal spheroids. The numbers of surviving Purkinje neurons were counted in the third cerebellar lobe from two adjacent sections for each animal and averaged, the length of the margin between the molecular and granule cell layers was measured, and the number of Purkinje neurons per millimeter was calculated. The number of surviving Purkinje neurons per millimeter was averaged within genotypes $\left(n=8 \mathrm{npc}^{-/-}\right.$and 8 $\left.n p c^{-\prime-}, p 35^{-1-}\right)$. For axonal spheroid quantitation, the numbers of axonal spheroids $8 \mu \mathrm{m}$ or larger in a $925 \times 740 \mu \mathrm{m}$ field were counted in anatomically matched sections of three brain regions: central pons, cerebellar peduncles, and inferior colliculus. For each animal, spheroids were counted in two adjacent sections and averaged. The average number of spheroids per animal was then averaged within genotypes $(n=6$ $n p c^{-1-}$ and $6 n p c^{-1-}, p 35^{-1-}$ ).

Motor ability and weight loss. Mice were weighed and motor ability was assessed twice weekly between 3 and 10 weeks of age. To assess motor ability, mice were allowed to grasp the bottom of a coat hanger suspended $18 \mathrm{~cm}$ above a flat surface and allowed to remain on the hanger for up to 2 min (hanging time). Their behavior on the hanger was also noted (see Fig. 4). Hanging time was multiplied by behavior to obtain a numeric 
value for motor ability. Values were averaged within genotypes at each time point ( $n=13 \mathrm{wt}, 14 \mathrm{p} 35^{-/-}, 10 \mathrm{np} \mathrm{c}^{-/-}$, and $\left.13 \mathrm{np} \mathrm{c}^{-/-}, \mathrm{p} 35^{-/-}\right)$.

Statistical analyses. All statistical analyses were performed using the Student's $t$ test. Significance is indicated at ${ }^{*} p<0.05$ or ${ }^{* *} p<0.001$. Sampling errors are reported as the SEM.

\section{Results}

Cdk5 activity is reduced in $n p c^{-/-}, p 35^{-/-}$mice

Cdk5 protein levels and activity were examined in supernatants from brain homogenates of $n p c^{-/-}$and $n p c^{-/-}, p 35^{-/-}$mice (Fig. 1). Western blotting detected similar levels of $\mathrm{cdk} 5$ protein in brain supernatants of both genotypes of mice using two different cdk5 antibodies (Fig. $1 A$, shown for DC17). In contrast to $n p c^{-/-}$mice, $\mathrm{p} 35$ and $\mathrm{p} 25$ were absent from $n p c^{-/-}, p 35^{-/-}$mice ( shown for $\mathrm{p} 25 \mathrm{~N}$ ). Levels of the cdk5 activator p39 were similar in both groups, ruling out compensatory upregulation attributable to lack of p35/p25.

In vitro cdk5 kinase assays detected a $78.2 \pm 8.9 \%$ reduction $(p<0.05)$ in cdk5 activity in $n p c^{-/-}, p 35^{-/-}$mice compared with $n p c^{-/-}$mice (Fig. $1 B$ ), indicating that much of the $c d k 5$ activity in $n p c^{-/-}$mice is mediated by $\mathrm{p} 35$ or p25. The residual cdk5 activity in $n p c^{-/-}, p 35^{-/-}$mice was similar to that of $p 35^{-/-}$ mice and $32.5 \pm 13.3 \%(p<0.05)$ that of $w t$ mice. The marked inhibition of cdk5 in $n p c^{-/-}, p 35^{-/-}$mice makes this model suitable for studying the role of p35/p25 and cdk5 in NPC.

Tau phosphorylation is not reduced in $n p c^{-/-}, p 35^{-/-}$mice Previous studies found increased tau phosphorylation at several different epitopes in the brains of $n p c^{-/-}$mice compared with $w t$ (Bu et al., 2002a), and administration of cdk inhibitors in $n p c^{-1-}$ mice reduced phosphorylation at these epitopes in a dose-dependent manner (Zhang et al., 2004). If p35/p25-mediated cdk5 activity is responsible for hyperphosphorylation in NPC, $n p c^{-1-}$ mice with greatly reduced cdk5 activity should have reduced phosphorylation.

We examined levels of tau and tau phosphoepitopes in $w t, n p c^{-/-}$, and $n p c^{-/-}, p 35^{-/-}$mouse brains (Fig. 2). We found similar levels of total tau (TG5) in $w t$ and $n p c^{-1-}$ mice, but levels were increased in $n p c^{-/-}, p 35^{-/-}$mice, consistent with previous findings in $p 35^{-/-}$mice (Hallows et al., 2003). After normalizing to TG5 immunoreactivity, tau phosphorylation in $n p c^{-1-}$ mice was significantly increased at Ser-202 (CP13) and Ser-396/ 404 (PHF-1) compared with wt. In $n p c^{-/-}, p 35^{-/-}$mice, tau phosphorylation at Ser-202 (CP13) was even further increased, almost threefold higher than $w t$ and twofold higher than $n p c^{-1-}$ mice. Tau phosphorylation at Ser-396/404 was increased almost twofold in $n p c^{-/-}, p 35^{-1-}$ mice compared with $w t$. These data demonstrate that hyperphosphorylation in $n p c^{-/-}$mice occurs independent of $\mathrm{p} 35 /$ p25-mediated cdk5 activation.

At the immunohistochemical level, CP13 and PHF-1 immunoreactivities were increased in $n p c^{-1-}$ mice compared with $w t$ mice and were localized to axons and axonal spheroids (Fig. $2 \mathrm{~B}$, shown for CP13 only). In $n p c^{-1-}$, $p 35^{-/-}$mice, CP13 immunoreactivity was qualitatively similar but more intense than in $n p c^{-/-}$mice in regions with cytoskeletal pathology (pons, left panels) and in the neuropil of regions without overt cytoskeletal pathology, i.e., the cortex (right panels).
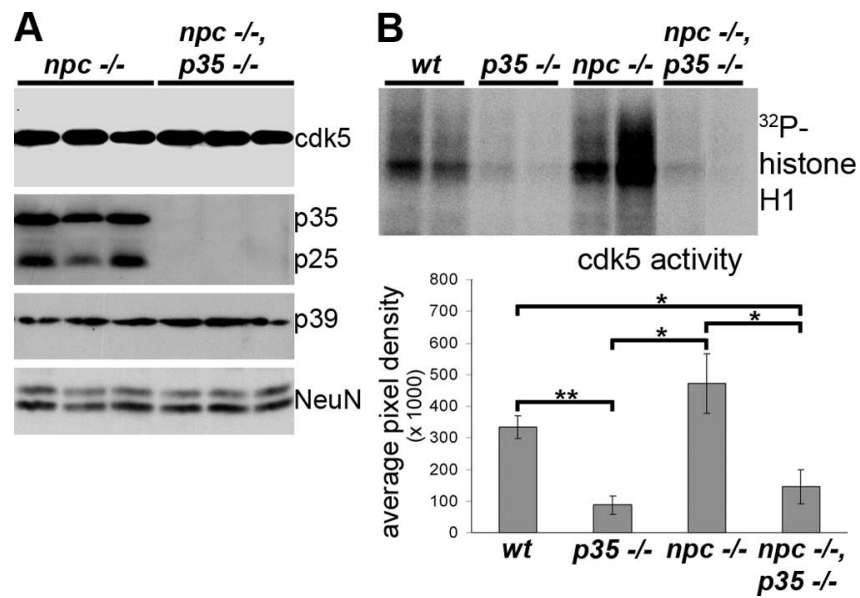

Figure 1. c cdk5 levels and activity. $A$, Western blots showing similar levels of cdk5 and p39 in $n p \mathrm{c}^{-/-}$and $n p \mathrm{c}^{-/-}, \mathrm{p35^{-/- }}$ mouse brains. $\mathrm{p} 35$ and $\mathrm{p} 25$ were detected in $n p \mathrm{c}^{-/-}$mice but not in $n p c^{-1-}, p 35^{-/-}$mice. NeuN is shown as a protein loading control. $\boldsymbol{B}$, Autoradiograph from an in vitro $c \mathrm{dk} 5$ kinase assay and graphic representation of the average $\mathrm{cdk} 5$ activity for each genotype. Cdk5 activity was reduced $\sim 78 \%$ in $n \mathrm{pc}^{-/-}, p 35^{-/-}$mice compared with $n \mathrm{pc}^{-1-}$ mice.
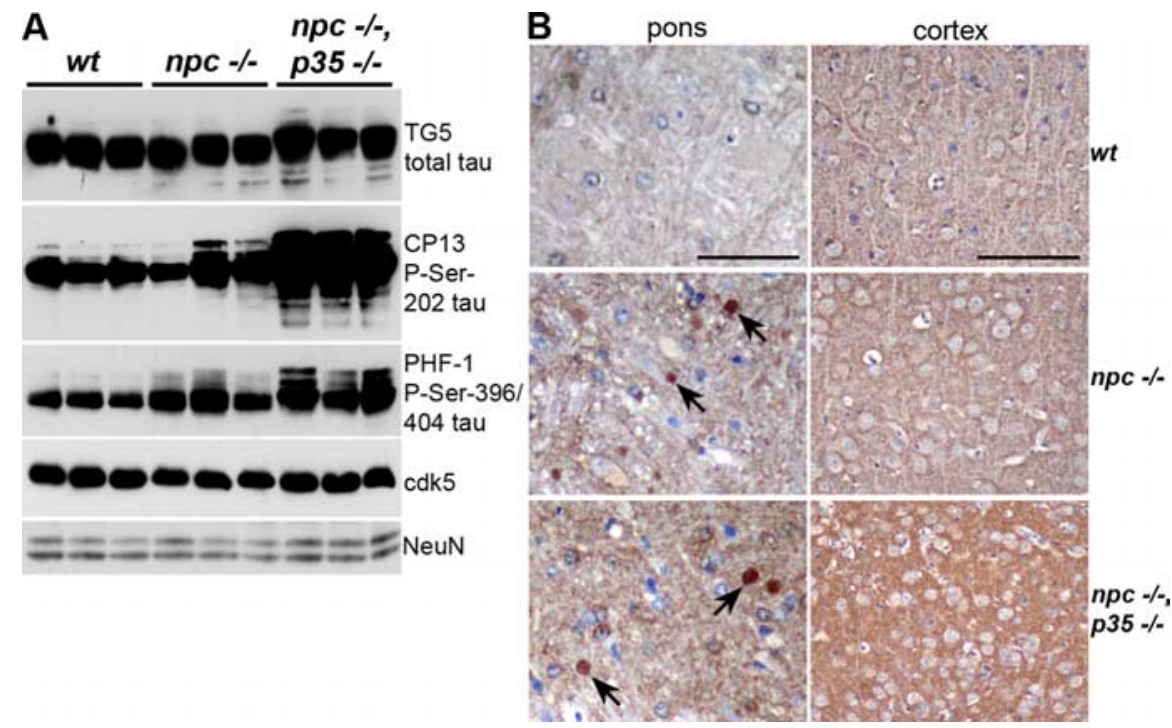

Figure 2. Tau phosphorylation. $\boldsymbol{A}$, Western blots showing similar levels of total tau (TG5) in $w t_{\text {and }} n p \mathrm{c}^{-/-}$mice but increased total tau $(107.4 \pm 40.4 \% ; p<0.05)$ in $n p c^{-/-}, p 35^{-/-}$mice compared with $w t$ and $n p c^{-/-}$mice. Tau phosphorylation at Ser-202 (CP13) was increased in $n p c^{-1-}$ mice $(106.7 \pm 37.6 \% ; p<0.05)$ and further increased in $n p c^{-1-}$, $p 35^{-1-}$ mice $(292.5 \pm 55.9 \% ; p<0.001)$ compared with wt or increased $90.4 \pm 27.1 \%(p<0.05)$ compared with $n p c^{-1-}$ mice. Tau phosphorylation at Ser-396/404 (PHF-1) was increased in both $n p c^{-1-}(68.5 \pm 31.7 \% ; p<0.05)$ and $n p c^{-/-}, p 35^{-1-}$ $(97.1 \pm 19.8 \% ; p<0.001)$ mice compared with $w$ t but was not significantly different between $n p c^{-/-}$and $n p c^{-1-}, p 35^{-1-}$ mice. Tau phosphorylation increased in both $n p c^{-/-}$and $n p c^{-/-}, p 35^{-/-}$mice despite similar levels of cdk5 in all three genotypes of mice. NeuN is shown as a protein loading control. $\boldsymbol{B}$, Sagittal brain sections stained with the tau phospho-Ser-202 antibody CP13 in $w t, n p c^{-/-}$, and $n p c^{-/-}, p 35^{-/-}$brains in the pons (left; scale bar, $50 \mu \mathrm{m}$ ) and cortex (right; scale bar, 100 $\mu \mathrm{m}) .\left(\mathrm{CP} 13\right.$ immunoreactivity was seen in axonal spheroids (arrows) in the pons in both $n p c^{-/-}$and $n p c^{-/-}, p 35^{-/-}$brains. Staining was more robust in $n p c^{-/-}$brains compared with wt and was even more robust in $n p c^{-1-}, p 35^{-/-}$brains compared with $n p c^{-/-}$brains. The disruption of the normal layered cytoarchitecture of the cortex seen in the $n p c^{-/-}, p 35^{-/-}$brain is a component of the $235^{-/-}$phenotype (Hallows et al., 2003). 

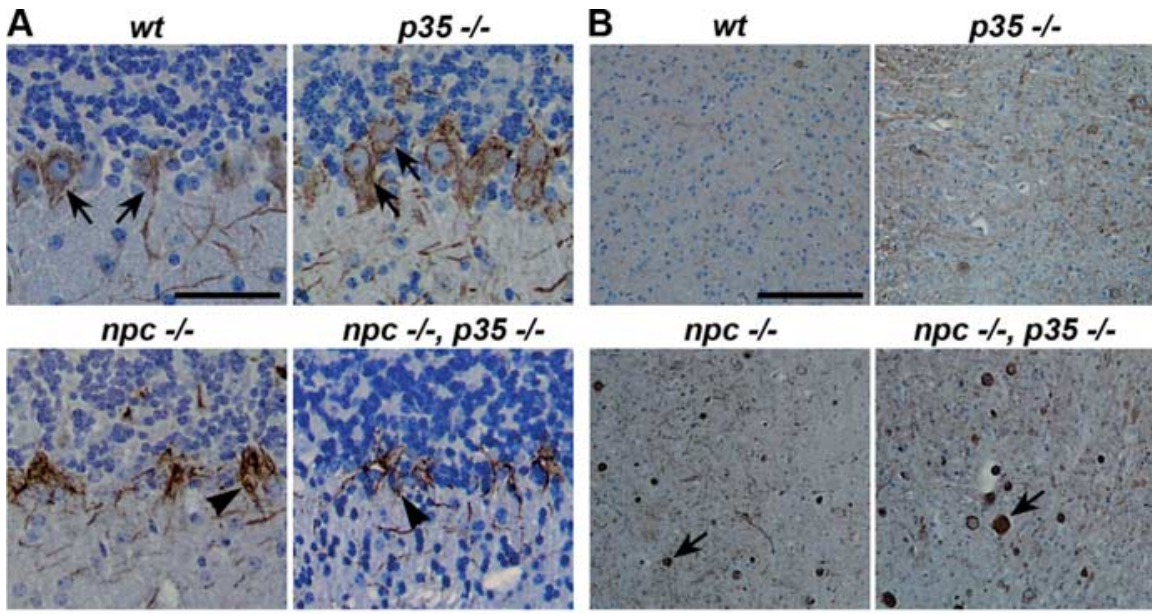

npc $\%-$, p35 -/-
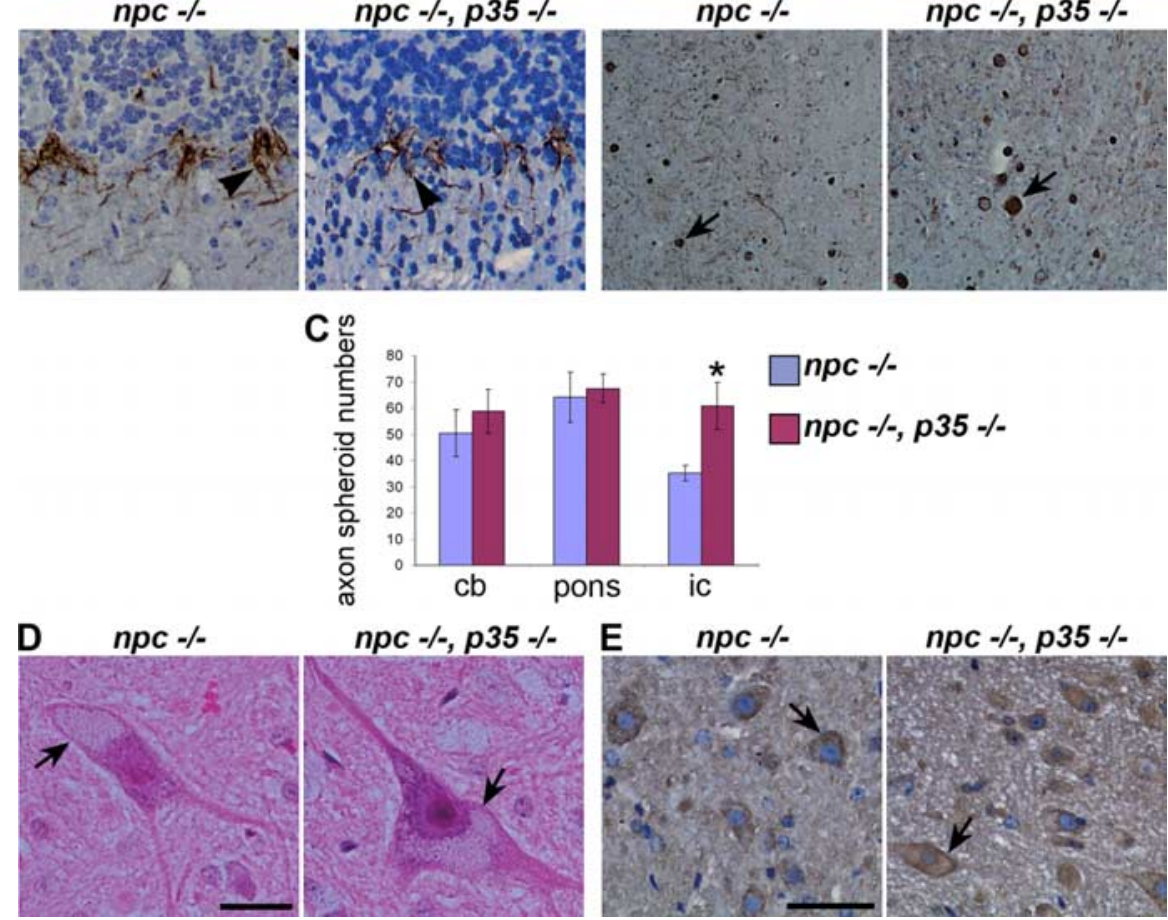

Figure 3. Pathological lesions. A, Sagittal brain sections stained with the NF antibody SMI32 showing normally (wt) and ectopically $\left(p 35^{-1-}\right.$ ) localized cerebellar Purkinje neurons (arrows). Many Purkinje neurons have been lost in $n p c^{-1-}$ and $n p c^{-/-}, p 35^{-/-}$mice, but labeled basket cell terminals still surround their former location (arrowheads). The ectopically localized granule cells in the molecular layer in the $n p c^{-/-}, p 35^{-/-}$is a component of the $p 35^{-/-}$phenotype (Hallows et al. 2003). Scale bar, $50 \mu \mathrm{m}$. B, Sagittal brain sections stained with the NF antibody SMI32 showing no axonal spheroids in wt or $\mathrm{p} 35^{-/-}$mouse brains but numerous spheroids (arrows) in the pons of $\mathrm{npc}^{-/-}$and $n \mathrm{nc}^{-/-}, \mathrm{p} 35^{-/-}$mice. Scale bar, $100 \mu \mathrm{m}$. C, Graph showing similar numbers of spheroids in the cerebellum (cb) and pons of $n p c^{-/-}$and $n p c^{-/-}, p 35^{-1-}$ mice. Spheroid numbers increased $\sim 73 \%$ in the inferior colliculus (ic) in $n p c^{-1-}, p 35^{-1-}$ mice compared with $n p c^{-1-}$ mice. $D$, Sagittal brain sections stained with H\&E showing similar lipid accumulation and formation of distended neurons in $n p c^{-1-}$ and $n p c^{-1-}$, $p 35^{-1-}$ mice (arrows). Scale bar, $25 \mu \mathrm{m}$. E, Sagittal brain sections stained with an antibody to ganglioside GM2 showing similar accumulation of GM2 in $n p \mathrm{c}^{-/-}$and $n p \mathrm{c}^{-/-}, \mathrm{p35^{-/- }}$ mice (arrows). Scale bar, $50 \mu \mathrm{m}$.

PHF-1 did not reveal any striking difference in $n p c^{-/-}, p 35^{-/-}$ mice compared with $n p c^{-/-}$mice (data not shown).

Other tau phosphoepitopes, i.e., Thr-175 (CP22), Thr-231 (CP10), Ser-235 (MC6), and Ser-409 (PG5), were also examined by Western blotting and immunohistochemistry. With both techniques, no significant changes were observed with these phosphoepitopes when $n p c^{-/-}, p 35^{-/-}$mice and $n p c^{-/-}$mice were compared (data not shown).

Collectively, these data demonstrate that tau hyperphosphorylation in $n \mathrm{pc}^{-1-}$ mice proceeds even in the presence of drastically reduced cdk5 activity.

\section{Neuropathological hallmarks are not reduced in $n p c^{-/-}$, p35 $5^{-/-}$mice}

The brains of $n p c^{-/-}$mice are characterized by the formation of several neuropathological lesions, including lipid accumulation within neurons, formation of axonal spheroids, and neuron loss, most prominently of cerebellar Purkinje cells (Walkley and Suzuki, npc $-/$, p35 \%-

2004). Continuous intracerebroventricular administration of cdk inhibitors in $n p c^{-/-}$ mice resulted in improved Purkinje neuron survival and decreased axonal spheroid formation (Zhang et al., 2004). If p25-mediated cdk5 activity is involved in this pathogenesis, it is expected that formation of these pathologies would be reduced in $n p c^{-1-}$ mice lacking $\mathrm{p} 35 / \mathrm{p} 25$.

The Purkinje neuron monolayer is normally situated between the molecular and granule cerebellar layers (Fig. 3A,wt). In p35 null mice Purkinje neurons are often misplaced throughout the granular layer and sometimes aggregate with a multilayered appearance, and granule cells are misplaced in the molecular layer (Hallows et al., 2003). This abnormal distribution of Purkinje cells is not seen in $n p c^{-/-}$mice but is observed in $n p c^{-/-}, p 35^{-/-}$mice (Fig. $3 A$ ) and thus appears to be related to the absence of p35. Therefore, SMI32, an NF antibody that stains Purkinje cells (Gotow and Tanaka, 1994), was used to identify and count Purkinje cells in $n p c^{-/-}$ and $n p c^{-/-}, p 35^{-/-}$mice. We found a similar number of Purkinje neurons remaining in $n p c^{-/-}$and $n p c^{-/-}, p 35^{-/-}$cerebellae, demonstrating that the lack of p35/p25 does not improve Purkinje neuron survival in $n p c^{-1-}$ mice.

Although both tau and NFs accumulate in spheroids, we found that SMI32 immunoreactivity (Fig. $3 B$ ) is a more reliable marker for detecting these lesions ( $\mathrm{Bu}$ et al., 2002a). This antibody recognizes a nonphosphorylated sequence in the heavy subunit of NF, which is not affected by cdk inhibitor treatment (Zhang et al., 2004). SMI32-positive spheroids were not detected in $w t$ or $p 35^{-1-}$ mice but were present in $n p c^{-/-}$and $n p c^{-/-}, p 35^{-/-}$ mice. Similar numbers were counted in the pons and cerebellum, but the inferior colliculus of $n p c^{-1-}, p 35^{-1-}$ mice had a $72.8 \pm 14.7 \%(p<0.05)$ increase over $n p c^{-/-}$mice (Fig. $\left.3 C\right)$.

NPC is primarily a lipid storage disease and is characterized by the formation of distended neurons containing nonesterified lipids and the ganglioside GM2 (Walkley and Suzuki, 2004). Although there is no reason presently to expect any change in lipid pathology by altering the function of $\mathrm{cdk} 5$, we examined lipid storage in $n p c^{-/-}$and $n p c^{-/-}, p 35^{-/-}$mouse brains by $\mathrm{H} \& \mathrm{E}$ staining and GM2 immunostaining. Procedures involved in processing paraffin-embedded tissue usually lead to significant extraction of lipids, but evidence for lipid storage is seen by the foamy appearance of lipid in distended neurons and its negative reaction to $\mathrm{H} \& \mathrm{E}$. These indices were similar in both groups of mice (Fig. 3D), as was GM2 immunoreactivity (Fig. 3E).
Weight loss and motor defects are not reduced in $n p c^{-/-}$, p35 $5^{-/-}$mice $n p c^{-1-}$ mice characteristically experience weight loss and a de- cline in motor ability beginning at $\sim 6$ weeks of age (Loftus et al., 

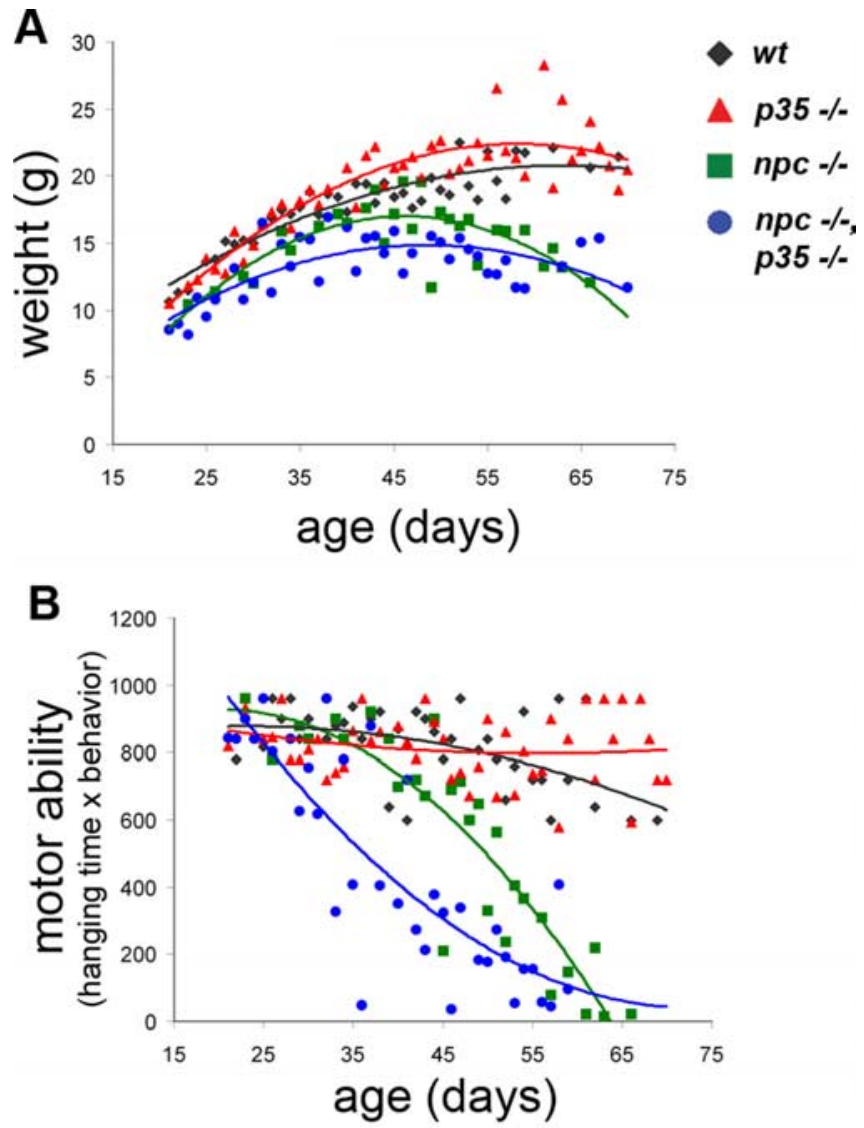

$\begin{array}{lc}\text { Behavior on coat hanger } & \text { value } \\ 1 \text { paw } & 1 \\ 2 \text { paws } & 2 \\ 3 \text { paws } & 3 \\ 4 \text { paws } & 4 \\ 4 \text { paws + tail or } 4 \text { paws balanced upright } & 5 \\ 4 \text { paws + tail, moved to end of hanger } & 6 \\ 4 \text { paws + tail, climbed onto slanted side } & 7 \\ 4 \text { paws + tail, climbed onto curved top } & 8\end{array}$

Figure 4. Weight and motor ability of $w t, p 35^{-1-}, n p c^{-1-}$, and $n p c^{-1-}, p 35^{-1-}$ mice between 3 and 10 weeks of age. $A, n p c^{-1-}$ and $n p c^{-1-}, p 35^{-1-}$ mice had similar weight loss. $\boldsymbol{B}$, Motor ability deteriorated in $n p c^{-/-}$mice beginning at $\sim 6$ weeks of age, whereas $n p c^{-1-}$, $p 35^{-/-}$mice exhibited a decline in motor ability beginning at $\sim 5$ weeks of age.

2002), and intracerebroventricular administration of cdk inhibitors in $n p c^{-1-}$ mice has been shown to improve both of these disease parameters (Zhang et al., 2004).

To determine whether weight loss or motor defects were improved in $n p c^{-/-}$mice lacking p35/p25, we weighed mice and tested their motor ability twice weekly between the ages of 3 and 10 weeks of age. Both $w t$ and $p 35^{-1-}$ mice continued to gain weight over the course of testing, whereas $n p c^{-/-}$and $n p c^{-/-}$, $p 35^{-/-}$mice lost weight beginning at $\sim 6$ weeks of age (Fig. $4 \mathrm{~A}$ ). Motor ability held fairly constant in $w t$ and $p 35^{-/-}$mice. $n p c^{-/-}$ mice demonstrated a dramatic decline in motor ability beginning at $\sim 6$ weeks of age, whereas $n p c^{-/-}, p 35^{-/-}$mice demonstrated this decline at an earlier time of $\sim 5$ weeks (Fig. $4 B$ ). Overall, these findings show no improvement in weight loss or motor ability in $n p c^{-/-}$mice lacking p35/p25.

\section{Discussion}

Our current study demonstrates that the cardinal features of NPC, i.e., tau hyperphosphorylation, axonal spheroid formation,
Purkinje neuron loss, weight loss, and motor defects, are not attenuated in $n p c^{-/-}, p 35^{-/-}$mice. Loss of p35/p25 not only negated the disease-associated elevation in cdk 5 activity but reduced it to $32 \%$ of $w t$ levels. The persistence of NPC pathology in the absence of $\mathrm{p} 35 / \mathrm{p} 25$ proves unequivocally that $\mathrm{p} 35 / \mathrm{p} 25$ mediated cdk 5 activation is not responsible for hyperphosphorylation and subsequent cytoskeletal pathology in NPC.

Ruling out cdk 5 entirely is more difficult. The $78 \%$ decrease in cdk5 activity in $n p c^{-/-}, p 35^{-/-}$mice, without an accompanying decrease in tau phosphorylation and axonal spheroid formation, suggests that cdk5 is not responsible for NPC pathology. Interestingly, phosphoepitopes thought to be produced by cdk5 [Ser231 and Ser-235 (Lew et al., 1994)] and the proline-directed Thr175 site were not affected; the CP13 Ser-202 site that can be produced by multiple proline-directed kinases was increased, and the PHF-1 epitope thought to be produced by glycogen synthase kinase $3 \beta$ (GSK3 $\beta$ ) (Lucas et al., 2001) was also increased. It could be argued that residual p35-independent cdk5 activity in $n p c^{-1-}, p 35^{-1-}$ mice may be sufficient to drive pathology. We have shown (Hallows et al., 2003) that loss of p35 in a wt background redistributes cdk 5 to more distal neuronal processes. This redistribution might concentrate $\mathrm{p} 35$-independent cdk5 activity to focal sites relevant to disease and improve its access to relevant substrates such as tau. In this scenario, the real culprit would be a lack or decreased levels of p35 rather than p25 accumulation or change in cdk5 activity. With respect to the latter, we (Hallows et al., 2003) and others (Sharma et al., 2002; Morfini et al., 2004) have proposed that cdk 5 negatively regulates downstream kinases that phosphorylate tau. According to this scheme, a decrease in p35 or reduced cdk5 activity would promote cytoskeletal pathology and neurodegeneration. It is likely that the kinases activated by cdk5 inhibition may be responsible for the increased tau hyperphosphorylation at the CP13 and PHF-1 epitopes in $n p c^{-/-}$, $p 35^{-1-}$ mice compared with $n p c^{-1-}$ mice. Because mice lacking cdk5 activity die at birth (Ohshima et al., 1996), a model of postdevelopmental cdk5 ablation would be more useful for testing these possibilities.

The negative effects of p35/p25 depletion were unexpected in light of the attenuating effects of cdk inhibitors in $n p c^{-/-}$mice (Zhang et al., 2004). Because roscovitine and olomoucine are not specific for cdk5, one explanation may be that other roscovitineand olomoucine-sensitive cdks are involved in NPC. In independent studies, we found that cell division cycle 2 (cdc2) and cdk4 are also activated in human NPC (Bu et al., 2002b) and $n p c^{-/-}$ mice (B. Bu and I. Vincent, unpublished data). These cdks are also present at early stages of AD (Vincent et al., 1997; Busser et al., 1998) and ALS (Nguyen et al., 2003). Roscovitine and olomoucine are most potent against cdc2 and cdk5 and essentially ineffective against cdk4 (Vesely et al., 1994; Meijer et al., 1997). Together with the present study casting doubts on $\mathrm{cdk} 5$, cdc2 may be the likely candidate.

An alternative but less likely explanation for the attenuating effects of cdk inhibitors in $n p c^{-/-}$mice is that other related kinases such as the extracellular-regulated kinases erk $1 / 2$ or GSK3 $\beta$ may be important. Roscovitine and olomoucine inhibit these kinases at $>10$-fold the $\mathrm{IC}_{50}$ for cdc2 (Meijer et al., 1997). The kinases have also been implicated in AD (Iqbal et al., 2005) and NPC (Sawamura et al., 2001). Although we showed that phosphorylation of known substrates for these kinases was not reduced by cdk inhibitors in $n p c^{-/-}$mice (Zhang et al., 2004), they cannot be ruled out entirely. $n p c^{-/-}$and $n p c^{-/-}, p 35^{-/-}$mice are suitable models for exploring these possibilities.

Our p35 null mice have been useful for examining the role of 
$\mathrm{cdk} / \mathrm{p} 25$ in other types of neurodegeneration. Induction of ischemic stroke in our $p 35^{-/-}$mice has shown that $\mathrm{cdk} 5 / \mathrm{p} 25$ is essential for the rapid or excitotoxic component of ischemic death but not the delayed or apoptotic component that requires cdk4 (Rashidian et al., 2005). Moreover, 1-methyl-4-phenyl-1,2,3,6tetrahydropyridine-induced calpain-mediated p25-dependent activation of cdk5, dopaminergic neuron loss, and behavioral deficits are attenuated in the $p 35^{-/-}$mice, indicating that $\mathrm{cdk} 5 /$ p25 is essential for Parkinson-type neurodegeneration (Smith et al., 2006). Conversely, the role of cdk5 and p25 in AD, ALS, and NPC remains controversial. Although some have reported increased levels of p25 in these diseases (Patrick et al., 1999; Nguyen et al., 2001; Bu et al., 2002a), others have found no increases (Yoo and Lubec, 2001; Tandon et al., 2003). At least three models of p25 overexpression have failed to result in hyperphosphorylation or relevant pathology (Ahlijanian et al., 2000; Takashima et al., 2001; Bian et al., 2002). Potential problems with overexpression models lie in the difficulties in matching activity levels to those observed in human diseases and in controlling the specific neuronal or intraneuronal distribution of increased activity. Moreover, increased cdk 5 or $\mathrm{p} 35$ expression does not accurately simulate AD, ALS, or NPC. In contrast, the $n p c^{-/-}$mouse model of neurodegeneration recapitulates the complexity of normal and pathological biochemical interactions in spatial and temporal aspects relevant to the disease. Our approach of targeted inhibition of specific effectors in such a model is a powerful means for molecular dissection of disease. Significantly, a similar approach using p35 deletion in the superoxide dismutase 1 transgenic mouse model of ALS also showed no effect on onset or progression of ALS (Takahashi and Kulkarni, 2004). These negative effects of p35/p25 depletion on NPC and ALS make it unlikely that p35/p25 would be essential for similar pathology in AD. The proposition that therapeutic management of these diseases might be achieved through targeted inhibition of cdk5 (Lau et al., 2002; Cruz and Tsai, 2004) needs to be reevaluated. The $n p c^{-1-}$ mouse offers the opportunity for investigating other kinases or players in these tau-associated pathologies.

\section{References}

Ahlijanian MK, Barrezueta NX, Williams RD, Jakowski A, Kowsz KP, McCarthy S, Coskran T, Carlo A, Seymour PA, Burkhardt JE, Nelson RB, McNeish JD (2000) Hyperphosphorylated tau and neurofilament and cytoskeletal disruptions in mice overexpressing human p25, an activator of cdk5. Proc Natl Acad Sci USA 97:2910-2915.

Axelsson R, Roytta M, Sourander P, Akesson HO, Andersen O (1984) Hereditary diffuse leucoencephalopathy with spheroids. Acta Psychiatr Scand Suppl 314:1-65.

Bian F, Nath R, Sobocinski G, Booher RN, Lipinski WJ, Callahan MJ, Pack A, Wang KK, Walker LC (2002) Axonopathy, tau abnormalities, and dyskinesia, but no neurofibrillary tangles in p25-transgenic mice. J Comp Neurol 446:257-266.

Bu B, Li J, Davies P, Vincent I (2002a) Deregulation of cdk5, hyperphosphorylation, and cytoskeletal pathology in the Niemann-Pick type C murine model. J Neurosci 22:6515-6525.

Bu B, Klunemann H, Suzuki K, Li J, Bird T, Jin LW, Vincent I (2002b) Niemann-Pick disease type $\mathrm{C}$ yields possible clue for why cerebellar neurons do not form neurofibrillary tangles. Neurobiol Dis 11:285-297.

Busser J, Geldmacher DS, Herrup K (1998) Ectopic cell cycle proteins predict the sites of neuronal cell death in Alzheimer's disease brain. J Neurosci 18:2801-2807.

Cruz JC, Tsai LH (2004) A Jekyll and Hyde kinase: roles for Cdk5 in brain development and disease. Curr Opin Neurobiol 14:390-394.

Cruz JC, Tseng HC, Goldman JA, Shih H, Tsai LH (2003) Aberrant Cdk5 activation by $\mathrm{p} 25$ triggers pathological events leading to neurodegeneration and neurofibrillary tangles. Neuron 40:471-483.

Delisle MB, Carpenter S (1984) Neurofibrillary axonal swellings and amyotrophic lateral sclerosis. J Neurol Sci 63:241-250.
Erickson RP, Bernard O (2002) Studies on neuronal death in the mouse model of Niemann-Pick C disease. J Neurosci Res 68:738-744.

German DC, Quintero EM, Liang CL, Ng B, Punia S, Xie C, Dietschy JM (2001) Selective neurodegeneration, without neurofibrillary tangles, in a mouse model of Niemann-Pick C disease. J Comp Neurol 433:415-425.

Gotow T, Tanaka J (1994) Phosphorylation of neurofilament H subunit as related to arrangement of neurofilaments. J Neurosci Res 37:691-713.

Hallows JL, Chen K, DePinho RA, Vincent I (2003) Decreased cyclindependent kinase 5 (cdk5) activity is accompanied by redistribution of cdk5 and cytoskeletal proteins and increased cytoskeletal protein phosphorylation in p35 null mice. J Neurosci 23:10633-10644.

Henderson LP, Lin L, Prasad A, Paul CA, Chang TY, Maue RA (2000) Embryonic striatal neurons from niemann-pick type $\mathrm{C}$ mice exhibit defects in cholesterol metabolism and neurotrophin responsiveness. J Biol Chem 275:20179-20187.

Iqbal K, Alonso Adel C, Chen S, Chohan MO, El-Akkad E, Gong CX, Khatoon S, Li B, Liu F, Rahman A, Tanimukai H, Grundke-Iqbal I (2005) Tau pathology in Alzheimer disease and other tauopathies. Biochim Biophys Acta 1739:198-210.

Lau KF, Howlett DR, Kesavapany S, Standen CL, Dingwall C, McLoughlin DM, Miller CC (2002) Cyclin-dependent kinase-5/p35 phosphorylates presenilin 1 to regulate carboxy-terminal fragment stability. Mol Cell Neurosci 20:13-20.

Lee VM, Goedert M, Trojanowski JQ (2001) Neurodegenerative tauopathies. Annu Rev Neurosci 24:1121-1159.

Lew J, Huang QQ, Qi Z, Winkfein RJ, Aebersold R, Hunt T, Wang JH (1994) A brain-specific activator of cyclin-dependent kinase 5. Nature 371:423-426.

Loftus SK, Morris JA, Carstea ED, Gu JZ, Cummings C, Brown A, Ellison J, Ohno K, Rosenfeld MA, Tagle DA, Pentchev PG, Pavan WJ (1997) Murine model of Niemann-Pick C disease: mutation in a cholesterol homeostasis gene. Science 277:232-235.

Loftus SK, Erickson RP, Walkley SU, Bryant MA, Incao A, Heidenreich RA, Pavan WJ (2002) Rescue of neurodegeneration in Niemann-Pick C mice by a prion-promoter- driven Npcl cDNA transgene. Hum Mol Genet 11:3107-3114.

Lucas JJ, Hernandez F, Gomez-Ramos P, Moran MA, Hen R, Avila J (2001) Decreased nuclear beta-catenin, tau hyperphosphorylation and neurodegeneration in GSK-3beta conditional transgenic mice. EMBO J 20:27-39.

Meijer L, Borgne A, Mulner O, Chong JP, Blow JJ, Inagaki N, Inagaki M, Delcros JG, Moulinoux JP (1997) Biochemical and cellular effects of roscovitine, a potent and selective inhibitor of the cyclin-dependent kinases cdc2, cdk2 and cdk5. Eur J Biochem 243:527-536.

Morfini G, Szebenyi G, Brown H, Pant HC, Pigino G, DeBoer S, Beffert U, Brady ST (2004) A novel CDK5-dependent pathway for regulating GSK3 activity and kinesin-driven motility in neurons. EMBO J 23:2235-2245.

Nguyen MD, Lariviere RC, Julien JP (2001) Deregulation of Cdk5 in a mouse model of ALS: toxicity alleviated by perikaryal neurofilament inclusions. Neuron 30:135-147.

Nguyen MD, Boudreau M, Kriz J, Couillard-Despres S, Kaplan DR, Julien JP (2003) Cell cycle regulators in the neuronal death pathway of amyotrophic lateral sclerosis caused by mutant superoxide dismutase 1. J Neurosci 23:2131-2140.

Noble W, Olm V, Takata K, Casey E, Mary O, Meyerson J, Gaynor K, LaFrancois J, Wang L, Kondo T, Davies P, Burns M, Veeranna, Nixon R, Dickson D, Matsuoka Y, Ahlijanian M, Lau LF, Duff K (2003) Cdk5 is a key factor in tau aggregation and tangle formation in vivo. Neuron 38:555-565.

Ohshima T, Ward JM, Huh CG, Longenecker G, Veeranna, Pant HC, Brady RO, Martin LJ, Kulkarni AB (1996) Targeted disruption of the cyclindependent kinase 5 gene results in abnormal corticogenesis, neuronal pathology and perinatal death. Proc Natl Acad Sci USA 93:11173-11178.

Patrick GN, Zukerberg L, Nikolic M, de la Monte S, Dikkes P, Tsai LH (1999) Conversion of $\mathrm{p} 35$ to $\mathrm{p} 25$ deregulates Cdk5 activity and promotes neurodegeneration. Nature 402:615-622.

Rashidian J, Iyirhiaro G, Aleyasin H, Rios M, Vincent I, Callaghan S, Bland RJ, Slack RS, During MJ, Park DS (2005) Multiple cyclin-dependent kinases signals are critical mediators of ischemia/hypoxic neuronal death in vitro and in vivo. Proc Natl Acad Sci USA 102:14080-14085.

Sawamura N, Gong JS, Garver WS, Heidenreich RA, Ninomiya H, Ohno K, Yanagisawa K, Michikawa M (2001) Site-specific phosphorylation of tau accompanied by activation of mitogen-activated protein kinase 
(MAPK) in brains of Niemann-Pick type C mice. J Biol Chem 276:10314-10319.

Sharma P, Veeranna, Sharma M, Amin ND, Sihag RK, Grant P, Ahn N, Kulkarni AB, Pant HC (2002) Phosphorylation of MEK1 by cdk5/p35 down-regulates the mitogen-activated protein kinase pathway. J Biol Chem 277:528-534.

Smith PD, Mount MP, Shree R, Callaghan S, Slack RS, Anisman H, Vincent I, Mao X, Park DS (2006) Calpain regulated p35/cdk5 plays a central role in dopaminergic neuron death through modulation of the transcription factor myocyte enhancer factor 2. J Neurosci 26:440-557.

Takahashi S, Kulkarni AB (2004) Mutant superoxide dismutase 1 causes motor neuron degeneration independent of cyclin-dependent kinase 5 activation by $\mathrm{p} 35$ or p25. J Neurochem $88: 1295-1304$.

Takashima A, Murayama M, Yasutake K, Takahashi H, Yokoyama M, Ishiguro K (2001) Involvement of cyclin dependent kinase5 activator p25 on tau phosphorylation in mouse brain. Neurosci Lett 306:37-40.

Tandon A, Yu H, Wang L, Rogaeva E, Sato C, Chishti MA, Kawarai T, Hasegawa H, Chen F, Davies P, Fraser PE, Westaway D, St. George-Hyslop PH (2003) Brain levels of CDK5 activator p25 are not increased in Alzhei- mer's or other neurodegenerative diseases with neurofibrillary tangles. J Neurochem 86:572-581.

Vesely J, Havlicek L, Strnad M, Blow JJ, Donella-Deana A, Pinna L, Letham DS, Kato J, Detivaud L, Leclerc S (1994) Inhibition of cyclin-dependent kinases by purine analogues. Eur J Biochem 224:771-786.

Vincent I, Jicha G, Rosado M, Dickson DW (1997) Aberrant expression of mitotic cdc2/cyclin B1 kinase in degenerating neurons of Alzheimer's disease brain. J Neurosci 17:3588-3598.

Walkley SU, Suzuki K (2004) Consequences of NPC1 and NPC2 loss of function in mammalian neurons. Biochim Biophys Acta 1685:48-62.

Yoo BC, Lubec G (2001) p25 protein in neurodegeneration. Nature 411: 763-764, discussion 764-765.

Zhang M, Li J, Chakrabarty P, Bu B, Vincent I (2004) Cyclin-dependent kinase inhibitors attenuate protein hyperphosphorylation, cytoskeletal lesion formation, and motor defects in Niemann-Pick type C mice. Am J Pathol 165:843-853.

Zhou L, Miller BL, McDaniel CH, Kelly L, Kim OJ, Miller CA (1998) Frontotemporal dementia: neuropil spheroids and presynaptic terminal degeneration. Ann Neurol 44:99-109. 